\title{
Leg Length Discrepancy in Cementless Total Hip Arthroplasty
}

\author{
Christopher N. Peck, Karan Malhotra, Winston Y. Kim \\ Department of Trauma and Orthopaedics, Salford Royal Hospital NHS Foundation Trust, \\ Manchester, Britain \\ E-mail: docpecker@hotmail.com \\ Received August 17, 2010; revised April 8, 2011; accepted April 18, 2011
}

\begin{abstract}
The use of cementless total hip arthroplasty (THA) is on the increase. In order to achieve rotational and axial stability larger implants may be required than originally templated for. This could potentially result in a larger leg length inequality. Our objective was to determine whether there is greater inequality in leg length post-operatively in cementless THA as compared to cemented implants. 136 consecutive patients undergoing elective THA between June 2007 and May 2008 were included. Post-operative digital radiographs were examined to determine leg length. Twenty seven patients (20\%) underwent a cemented procedure and 109 $(80 \%)$ a cementless procedure. In the cemented group the mean leg length discrepancy was $7.3 \mathrm{~mm}$ (range $19 \mathrm{~mm}$ short to $21 \mathrm{~mm}$ long). In the cementless group the mean measured leg length discrepancy was $6.3 \mathrm{~mm}$ (range $18 \mathrm{~mm}$ short to $23 \mathrm{~mm}$ long). There was no significant difference between the two groups $(\mathrm{P}=0.443)$. This study shows that with accurate pre-operative templating, both cemented and cementless procedures produce comparable and acceptable leg length discrepancies.
\end{abstract}

Keywords: Hip, Arthroplasty, Cementless, Leg Length

\section{Introduction}

Total hip arthroplasty is a very successful operation in the management of end stage hip osteoarthritis with over 64,000 primary procedures being performed in the UK last year [9]. Leg length discrepancy (LLD) is a well documented complication of total hip replacement. It has been shown to correlate strongly with patient dissatisfaction [5,8,11,17] and is a leading cause of litigation [4]. Reports in the literature show a change in leg length following THA from $21 \mathrm{~mm}$ short to $35 \mathrm{~mm}$ long $[5,7,15]$. Studies have shown that a discrepancy greater than 10 $\mathrm{mm}$ can result in a limp, back pain, sciatica, stiffness, hip dislocation, the need for a shoe raise and early failure $[7,13,15,17]$. These problems are particularly problematic in the younger population where the impact on quality of life may be more marked.

Cementless hip replacements are becoming increasingly popular with the perceived advantages of increased longevity and easier revision procedures. A potential disadvantage of a cementless system is that to achieve rotational and axial stability larger implants may be required intra-operatively than originally templated for.
This may result in inadvertent leg lengthening. The converse may occur if stability is achieved with a smaller implant. The use of a modular implant system means that the surgeon can make adjustments to the final leg length if necessary but this may affect the overall stability of the joint.

The aim of this study was to determine if leg length discrepancy exists to a greater degree in cementless THA compared with cemented hip replacements.

\section{Patients and Methods}

This retrospective study was performed at a single institution, a large University Teaching Hospital in the North-West of England. All surgeries were carried out by one of five lower limb arthroplasty consultants. The inclusion criteria were all patients undergoing a primary elective total hip arthroplasty (THA) between June 2007 and May 2008. Patients were excluded if the contralateral hip had already been replaced.

Preoperative templating was done using an overlay technique on hard film copies of digital radiographs. The size of the acetabular cup was templated first and its cen- 
tre of rotation marked. Using this, the size of the femoral stem was determined and the proposed neck cut that would best reproduce the normal neck-shaft angle and offset. All procedures were carried out under either a general or spinal anaesthetic and were performed with the patient in the lateral position through a posterior approach. Leg length was assessed intra-operatively by comparing the relative position of the knees through the drapes and the soft tissue tension during trial reduction. The cemented femoral implants used were the collarless polished taper (CPT) (Zimmer, Swindon, UK) and the Exeter stem (Stryker, Howmedica Osteonics, Berkshire, UK). The cementless femoral implants used were the Zimmer M/L Taper Hip Prosthesis with standard and extended offsets and modular heads and the CLS Zimmer cementless stem (Zimmer, Swindon, UK).

All post-operative radiographs were in the digital format on the Patient Archive and Communication System (PACS, General Electric) and measurements were made using the systems integrated measurement tools. Antero-posterior digital radiographs of the pelvis, centred on the pubic symphysis with the hips internally rotated $15^{\circ}$ were taken on the first post-operative day, prior to mobilisation. Limb length inequality was calculated using the method described by Woolson et al. [16] and subsequently by a number of other authors $[5,8,11,12,15]$. This is measured as the perpendicular distance from the inter-teardrop line to the most prominent point on the lesser trochanter of the femur. The distance on the post-operative side was compared to the contralateral limb which was assumed to be equal to the initial length of the operated limb before development of joint disease. Magnification was adjusted for by using a standard estimation of $20 \%$ magnification consistent with the literature $[3,10,14,15]$. All measurements were made on the initial post-operative radiographs and were made to the nearest millimetre by a single observer not involved in the surgical procedures. Each measurement was taken twice, at the same sitting, and an average value used to reduce intra-observer errors.

All data was analysed using Excel for Windows (Microsoft). Continuous parametric data were analysed using the two sample T-test and nonparametric data were analysed using the chi squared test $(\mathrm{p}<0.05$ was considered as significant).

\section{Results}

Between June 2007 and May 2008166 patients underwent a primary total hip arthroplasty (THA). Thirty patients had a pre-existing contralateral hip replacement and so were excluded. Of the 136 patients in the study 27 (20\%) underwent a cemented THA and 109 (80\%) a ce- mentless procedure. Fifty eight (43\%) patients were male and 78 (57\%) were female. The mean age of patients was 66.7 years in the cemented group and 66.4 in the cementless (Table 1).

Post-operatively in the cemented group seven patients (26\%) had some degree of radiographic shortening, 19 (74\%) had some degree of lengthening and one patient (4\%) had equal leg lengths. In the cementless group 32 patients (29\%) had some degree of radiographic shortening, 67 (61\%) had some degree of lengthening and 10 patients (9\%) had equal leg lengths. There was no significant difference between these proportions $(\mathrm{P}=$ 0.949).

When corrected for an estimated magnification of $20 \%$ the mean radiographic limb length inequality regardless of direction was $7.3 \mathrm{~mm}$ in the cemented group and $6.3 \mathrm{~mm}$ in the cementless group $(\mathrm{P}=0.496)$. In the cemented group two patients (7\%) had shortening greater than $10 \mathrm{~mm}$ (mean $16.5 \mathrm{~mm}$ ) and four patients (15\%) had lengthening greater than $10 \mathrm{~mm}$ (mean $17.3 \mathrm{~mm}$ ). In the cementless group five patients (5\%) had shortening greater than $10 \mathrm{~mm}$ (mean $14.0 \mathrm{~mm}$ ) and 17 patients (16\%) had lengthening greater than $10 \mathrm{~mm}$ (mean $14.0 \mathrm{~mm}$ ). There was no significant difference between these groups ( $\mathrm{P}=0.331$ for shortening, $\mathrm{P}=0.140$ for lengthening). These results are summarised in Table 1.

\section{Discussion}

To be successful, hip replacement surgery needs to accurately reconstruct the patient's own anatomy and biomechanics. This includes reproducing the centre of rotation of the hip joint, the offset and leg length. This requires the appropriate selection and orientation of implants and if not done appropriately will lead to post operative complications and patient dissatisfaction.

Leg length discrepancy can have a significant impact on patient satisfaction and is one of the leading causes of the litigation following THA in America [4,7]. Leg lengthening is much less tolerated than leg shortening, with lengthening over $10 \mathrm{~mm}$ being very poorly tolerated by the patient [16]. Lengthening of the operated limb can lead to joint stiffness, sciatic nerve palsy, low back pain, early failure of the prosthesis and the need for revision surgery [7,13,15,17]. Shortening can impair hip abduction and increase the risk of dislocation [13].

In a study of 75 patients Konyves et al. [5] showed that the post-operative Oxford Hip Score was significantly worse in patients with leg lengthening compared to those with equal leg lengths. Most other studies confirm that a discrepancy in leg length has a direct adverse effect on clinical and functional outcome $[8,11,17]$. In contrast, White et al. [15] showed no statistically significant 
Table 1. Patient demographics and post-operative radiographic leg length discrepancy (LLD) in cemented and cementless THA. (SD Standard Deviation).

\begin{tabular}{cccc}
\hline & Cemented THA & Cementless THA & Significance \\
\hline Number of Patients & $27(20 \%)$ & $109(80 \%)$ & \\
Mean Age (Range) & 66.7 years $(30-87)$ & 66.4 years (37 - 88) & $\mathrm{p}=0.930$ \\
Males (M)/Females (F) & $\mathrm{M}=15(56 \%)$ & $\mathrm{M}=43(39 \%)$ & \\
Overall mean LLD (SD) & $\mathrm{F}=12(44 \%)$ & $\mathrm{F}=66(61 \%)$ & $\mathrm{p}=0.496$ \\
Mean (SD) Shortened LLD [Range] & $7.3 \mathrm{~mm}(6.0)$ & $6.3 \mathrm{~mm}(4.9 \mathrm{~mm})$ & $\mathrm{p}=0.956$ \\
& $6.8 \mathrm{~mm}(7.2)$ & $6.8 \mathrm{~mm}(4.0)$ & $\mathrm{p}=0.475$ \\
\hline
\end{tabular}

difference in the Harris Hip Score and SF 36 Health Survey in patients with radiological leg lengthening or shortening. However, their outcome scores were measured at six months after surgery so long-term problems were not assessed. Furthermore it has been suggested that the Harris Hip Score may not fully take into account the patients' subjective experience of pain [17]. The position of the acetabular component has a much smaller impact on leg length, it has been shown that only $2 \%$ of patients with a leg length discrepancy were attributed to the position of the acetabular component [5].

True and apparent leg length should be measured preoperatively in all patients to guide the surgeon as to how the joint should be reconstructed [7]. Intra-operatively, with the patient in the lateral position, leg length can be judged by comparing the relative knee and ankle position and by assessment of soft tissue tension. However, this is not always accurate as any small change in the position of the patient can lead to a big change in apparent leg length and be misleading $[11,16]$. An additional part of the preoperative plan is hip templating which is routinely used to guide the surgeon towards the size and placement of implants, the neck length, offset, level of the femoral osteotomy and the restoration of limb length required. This can be done by the traditional overlay method or by using a computer package for digital radiographs. These methods have been shown to have variable correlation with the actual implants used [2,14] and so may be deceptive to the surgeon leading to difficulty in achieving equal leg lengths.

Despite evidence of better short to medium term results for cemented implants there is an increasing tendency towards cementless fixation with the perceived advantages of increased longevity and easier revision procedures. In 2008 33\% of implants were cementless compared to $21 \%$ in 2004 and this trend looks set to continue [9]. With cementless hip replacements rotational and axial stability is achieved intra-operatively by a press fit technique. We felt that in many cases a larger implant was required to accomplish this than was originally templated for with the possibility of inadvertently causing leg length discrepancy. The majority of cementless components increase in offset with increasing component size and therefore lengthen the limb. Modular implant systems may allow the surgeon to make adjustments to the final leg length if necessary but this may affect the overall stability of the joint. Final adjustments would be impossible with a mono-block system. In a laboratory study Barink et al. [1] assessed how well two different cementless hip systems matched the final rasp position. Using synthetic and cadaveric femurs the average rasp-stem mismatch was within $2 \mathrm{~mm}$ in three different planes, the mismatch being larger in the cadaveric femurs. The authors felt that this mismatch was of low clinical relevance. However, any mismatch may be misleading when performing a trial reduction to determine the final implant size. As far as we are aware this study has not been repeated with other cementless stems leaving any mismatch with these systems unknown.

There are very few clinical or radiological studies in the literature evaluating leg length discrepancy in cementless hip replacements. In a radiographic analysis Leonard et al. [6] compared cemented and cementless hip replacements for offset and limb length. They showed that cementless procedures resulted in a greater degree of leg lengthening with a mean of $5.6 \mathrm{~mm}$ compared to $3.8 \mathrm{~mm}$ in the cemented group, this was statistically significant. Our results show an overall mean leg length discrepancy of $7.3 \mathrm{~mm}$ in the cemented group and $6.3 \mathrm{~mm}$ in the cementless group which is consistent with other published series [5,7,15]. In contrast to Leonard et al. [6] we found no significant difference in radiographic leg length discrepancy between cementless and cemented implants. This may simply be due to the spread of our results shown by the high standard deviations, possibly due to the five different surgeons per- 
forming the hip replacements. However, no measure of spread was provided in Leonard's paper.

We acknowledge the limitations of the present study which should be considered when interpreting the results. The surgeries were carried out by five different consultants in lower limb reconstruction each with their own variations on templating and intraoperative methods to equalise leg lengths. No account was taken of the pre-existing limb length which can have a direct impact on the post operative limb length. Additionally, no assessment was made of disease in the contralateral hip which may also have a bearing on a surgeons desired final leg length of the operated hip. Due to the retrospective nature of the study we were unable to compare the size of the prosthesis used with the size of prosthesis templated for. This would have given us a better understanding as to whether the size of final implants may have changed leg length.

The general limitations of using radiographs to make our measurements include the variation in positioning of the pelvis with respect to the x-ray film and variations in relative magnification due to distance from the film. However, as these factors affect both hips and we are comparing the difference between the two it is reasonable to use plain radiographs to assess leg length. Our methods of measuring leg length discrepancy have been used in numerous other studies [5,8,11,12,15] and are considered to be accurate and reliable. To calculate the magnification the radiographic diameter of the prosthetic femoral head can be compared to the actual diameter of the prosthesis. This data was not available in the current study so a standard magnification factor of $20 \%$ was used to adjust the actual measurements taken from the radiographs. However, this is the standard magnification used in most templating systems and consistent with other studies $[3,10,14,15]$.

\section{Conclusions}

In conclusion, total hip arthroplasty is a safe, effective and reproducible treatment for end stage degenerative hip disease. The current trend is towards the use of cementless implants particularly in the younger patient. Our study shows that there is no significant difference in leg length discrepancy between cementless and cemented implants. This suggests that with accurate pre-operative templating both cemented and cementless procedures produce comparable and acceptable leg length discrepancies.

\section{Conflict of Interest Statement}

No benefits in any form have been received or will be received from a commercial party related directly or indirectly to the subject of this article.

\section{References}

[1] M. Barink, H. Meuers, M. Spruit, C. F. Fankhauser and N. Verdonschot, "How Close Does an Uncemented Hip Stem Match the Final Rasp Position?,” Acta Orthopaedica Belgica, Vol. 70, No. 6, 2004, pp. 534-539.

[2] L. W. Carter, D. O. Stovall and T. R. Young, "Determination of Accuracy of Preoperative Templating of Noncemented Femoral Prostheses," The Journal of Arthroplasty, Vol. 10, No. 4, 1995, pp.507-513. doi:10.1016/S0883-5403(05)80153-6

[3] K. S. Conn, M. T. Clarke and J. P. Hallett, "A Simple Guide to Determine the Magnification of Radiographs and to Improve the Accuracy of Preoperative Templating,” Journal of Bone and Joint Surgery, Vol. 84-B, No. 2, 2002, pp. 269-272. doi:10.1302/0301-620X.84B2.12599

[4] A. A. Hofmann and M. C. Skrzynski, "Leg Length Inequality and Nerve Palsy in Total Hip Arthroplasty: A Lawyer Awaits,” Orthopaedics, Vol. 23, No. 9, 2000, pp. 943-944.

[5] A. Konyves and G. C. Bannister, "The Importance of Leg Length Discrepancy after Total Hip Arthroplasty,” Journal of Bone and Joint Surgery, Vol. 87-B, No. 2, 2005, pp. 155-157.

[6] M. Leonard, P. Magill, P. Kiely and G. Khayyat, "Radiographic Comparison of Cemented and Uncemented Total Hip Arthroplasty and Hip Resurfacing,” European Journal of Orthopaedic Surgery and Traumatology, Vol. 17, No. 6, 2007, pp. 583-586. doi:10.1007/s00590-007-0228-y

[7] W. J. Maloney and J. A. Keeney, "Leg Length Discrepancy after Total Hip Arthroplasty," The Journal of Arthroplasty, Vol. 19, No. 4, 2004, pp. 108-110. doi:10.1016/j.arth.2004.02.018

[8] S. B. Murphy and T. M. Ecker, "Evaluation of a New Leg Length Measurement Algorithm in Hip Arthroplasty," Clinical Orthopaedics and Related Research, Vol. 463, 2007, pp. 85-89.

[9] National Joint Registry 6th Annual Report, 2009. http://www.njrcentre.org.uk/njrcentre/linkclick.aspx?fileti cket=euukur4jpyc\%3d\&tabid=86\&mid=523

[10] M. J. Oddy, M. J. Jones, C. J. Pendergrass, J. R. Pilling and J. A. Wimhurst, "Assessment of Reproducibility and Accuracy in Templating Hybrid Total Hip Arthroplasty Using Digital Radiographs,” Journal of Bone and Joint Surgery, Vol. 88, No. 5, 2006, pp. 581-585.

[11] C. S. Ranawat, R. R. Rao, J. A. Rodriguez and H. S. Bhende, "Correction of Limb-Length Inequality During Total Hip Arthroplasty," The Journal of Arthroplasty, Vol. 16, No. 6, 2001, pp. 715-721. doi:10.1054/arth.2001.24442

[12] N. Sugano, T. Nishii, H. Miki, H. Yoshikawa, Y. Sato, and S. Tamura, "Mid-Term Results of Cementless Total 
Hip Replacement Using a Ceramic on Ceramic Bearing with and Without Computer Navigation," Journal of Bone and Joint Surgery, Vol. 89, No. 4, 2007, pp. 455-460.

[13] K. T. Suh, S. J. Cheon and D. W. Kim, "Comparison of Preoperative Templating with Post-Operative Assessment in Cementless Total Hip Arthroplasty," Acta Orthopaedica Scandinavica, Vol. 75, No. 1, 2004, pp. 40-44. doi:10.1080/00016470410001708070

[14] A. Unnanuntana, D. Wagner and S. B. Goodman, "The Accuracy of Preoperative Templating in Cementless Total Hip Arthroplasty,” The Journal of Arthroplasty, Vol. 24, No. 2, 2009, pp. 180-186. doi:10.1016/j.arth.2007.10.032

[15] T. O. White and T. W. Dougall, "Arthroplasty of the Hip
Leg Length Is Not Important,” Journal of Bone and Joint Surgery, Vol. 84-B, No. 3, 2002, pp. 335-338. doi:10.1302/0301-620X.84B3.12460

[16] S. T. Woolson, J. M. Hartford and A. Sawyer, "Results of a Method of Leg-Length Equalization for Patients Undergoing Primary Total Hip Replacement,” The Journal of Arthroplasty, Vol. 14, No. 2, 1999, pp. 159-164. doi:10.1016/S0883-5403(99)90119-5

[17] V. Wylde, S. L. Whitehouse, A. H. Taylor, G. C. Bannister and A. W. Blom, "Prevalence and Functional Impact of Patient-Perceived Leg Length Discrepancy after Hip Replacement," International Orthopaedics, Vol. 33, No. 4, 2009, pp. 905-909.

doi:10.1007/s00264-008-0563-6 